\title{
Frege sobre "decisiones" (Entschlüsse): intencionalidad y motivación en Frege y Husserl
}

\author{
Frege on "decisions" (Entschlüsse): intentionality and motivation by \\ Frege and Husserl
}

\section{Mario Ariel González Porta}

Doctor en filosofía por la Westfälische Wilhelms-Universität Münster, profesor titular de la Pontificia Universidad Católica de São Paulo (PUC-SP), São Paulo, SP - Brasil, e-mail: mariopor@pucsp.br

\section{Resumen}

Existe en Frege no solo una concepción intencional de la conciencia, sino también una concepción motivacional de su causalidad, esto es, Frege concibe la idea de una causalidad específica, diferente de la científico-natural, y que tiene como presupuesto la existencia de estados intencionales. En tal sentido, existe un interesante punto de contacto entre Frege y Husserl.

Palabras-clave: Intencionalidad. Motivación. Frege. Husserl. 
GONZÁLEZ PORTA, M. A.

\section{Abstract}

There is in Frege not only an intentional conception of consciousness, but also a motivational conception of its causality, i.e., Frege conceives the idea of a specific causality that differs from the one from the natural sciences and that presupposes the existence of intentional states. Regarding this matter, there is one more interesting contact point between Frege and Husserl.

Keywords: Intentionality. Motivation. Frege. Husserl.

L (1897) - Logik.

G - Der Gedanke.

GA - Die Grundlagen der Arithmetik.

GGA - Grundgesetze der Arithmetik. Begriffschriftlich abgeleitet.

Existe, al día de hoy, una lectura estándar en lo referente a la relación de Frege con temas que impliquen la consideración de la subjetividad. Esta interpretación se deja resumir en cuatro tesis:

a) Frege remite el problema del cómo de la aprehensión del pensamiento (Gedanke) a la psicología;

b) Frege no tiene interés alguno por problemas referentes a la subjetividad;

c) Frege identifica, sin más, psicología y psicología científico-natural;

d) Frege posee una concepción "naturalística" de la subjetividad".

La interpretación estándar puede asumir una forma moderada o una radical, según se limite a afirmar las dos primeras tesis o afirme, así mismo, la tercera y la cuarta. Característico de la forma radical es que ella no solo suscribe las tesis b y d, sino que considera que las mismas se siguen necesariamente de a y $\mathrm{c}$.

\footnotetext{
1 A los efectos de la exactitud hago uso de la distinción husserliana entre "naturalista" y "naturalístico". No cabe dudas que, en tanto no efectúa la reducción trascendental, la concepción fregueana de la subjetividad es naturalista. Por razones puramente lingüísticas continúo valiéndome de la expresión "naturalismo".
}

Rev. Filos., Aurora, Curitiba, v. 25, n. 37, p. 289-303, jul./dez. 2013 
Desde hace ya algún tiempo hemos intentado tornar plausible una lectura alternativa a la estándar. Para ello, consideramos como fuera de cuestión que, de hecho, Frege afirma a y c; cuestionamos, no obstante, que sea lícito atribuirle también tanto $b$ cuanto $d y$, más aún, que b y d se deriven necesariamente de a y c. En tal sentido, hemos defendido la tesis que

-b. Frege dispone de una "teoría de la subjetividad";

$-\mathrm{d}^{\prime}$. la cual concibe esta de un modo intencional;

$-\mathrm{d}^{\prime \prime}$. siendo que esta concepción intencional tiene como carácter diferencial el hecho de que niega expresamente toda forma del principio de inmanencia.

Podría cuestionarse nuestra lectura, diciéndose que, aun cuando no cabe duda de que Frege niega la tesis que mis únicos objetos inmediatos son mis representaciones, no obstante, esta negación, así como las parcas observaciones que la acompañan, están lejos de justificar el atribuirle pomposamente algo así como una "teoría de la subjetividad"2. Por esa razón es importante observar todos aquellos puntos que acompañan la anteriormente mencionada afirmación - $\mathrm{d}^{\prime}$ y constituyen un todo unitario con la misma. Existen, en efecto, otras doctrinas en el texto fregueano, que son coherentes con la concepción intencional no-inmanentista básica y que la complementan armoniosamente en aspectos particulares. Entre ellas hemos indicado, en otro momento, la teoría de la percepción y del pensamiento ${ }^{3}$. En este texto me propongo abordar otra, a saber, la idea de un tipo particular de causalidad que es propia de estados intencionales.

\section{Frege y el naturalismo: la aceptación de la psicología asociacionista}

Ya que el propósito de la presente contribución es aportar nuevas evidencias en el sentido de que Frege tiene una concepción intencional

\footnotetext{
2 Confieso que la expresión "teoría de la subjetividad" sugiere más de aquello que Frege efectivamente ofrece y, por tal razón, en otros momentos he optado por hablar de una "idea" 0 "concepción" de la subjetividad o de "tesis que pertenecen a una teoría de la subjetividad". En el presente texto, no obstante, y por razones de espacio, no entraré en esas distinciones. Véase nuestro artículo "La lógica fregueana de 1897 y su réplica al psicologismo" (de próxima aparición).

3 Compárese GONZÁLEZ PORTA, 2000.
} 
y no-naturalística de la subjetividad, es importante subrayar desde el comienzo, que hay, sin duda, elementos que inducen a pensar lo contrario y, en tal sentido, sustentan la tesis d:

i. Cuando Frege habla de la psicología, presupone siempre que esta es una ciencia natural. En ningún momento parece considerar la posibilidad de otra psicología;

ii. No solo de forma implícita, sino también explícita, Frege proporciona base a la tesis referida, en cuanto afirma que el objeto de la psicología es el establecimiento de un sistema de leyes que den cuenta del acontecer psíquico (G, 58(30));

iii. Mas aún, Frege identifica en reiteradas ocasiones las leyes psicológicas con las leyes de la asociación, lo cual lleva a pensar que él admite sin cuestionar el asociacionismo (GA).

Estas tres tesis son coherentes entre sí y, en cierta forma, se exigen recíprocamente; en las tres, no obstante, de una forma u otra, se pacta con la naturalización de la conciencia.

\section{Decisiones (Entschlüsse) y representaciones (Vorstellungen): un curioso texto y su interpretación}

Existe un curioso texto en "Der Gedanke" (que hasta ahora, por regla general, ha sido pasado por alto ${ }^{4}$ ) en el cual se observa que decisiones no son representaciones ${ }^{5}$. La afirmación de Frege es absolutamente inequívoca; la pregunta obvia es, entonces, ¿por qué?

La respuesta correcta me parece que es la siguiente:

4 Una excepción la constituye Künne (2010), quien en un trabajo reciente percibe que la observación freguena es, por lo menos, curiosa y, sobre tal base, considera un par de posibles interpretaciones; permanece no obstante indeciso, admitiendo que ninguna de ellas es plenamente satisfactoria.

5 "Auch der unphilosophische Mensch sieht sich bald genötigt eine, von der Aussenwelt verschiedene Innenwelt anzuerkennen, eine Welt der Sinneseindrücke, der Schöpfungen seiner Einbildungskraft, der Empfindungen, der Gefühle und Stimmungen, eine Welt der Neigungen, Wünsche und Entschlüsse. Um einen kurzen Ausdruck zu haben, will ich dies mit Ausnahme der Entschlüsse unter dem Worte 'Vorstellung' zusammenfassen" (G, 66(39)). (Todos los subrayados en las citas de Frege son míos).

Rev. Filos., Aurora, Curitiba, v. 25, n. 37, p. 289-303, jul./dez. 2013 
a) Representaciones son aquello que se encuentra sometido a leyes naturales, más concretamente, a las leyes de la asociación;

b) Decisiones, sin embargo, no pueden estar sometidas razonablemente a las leyes de la asociación, pues, si lo estuviesen, siempre estarían causalmente determinadas y;

c) en consecuencia, se debería sacrificar toda posibilidad de libertad;

d) lo cual tiene repercusiones obvias tanto en el plano práctico como en el teórico.

Dado que se podría conceder que lo expuesto es altamente plausible y, no obstante, llamar la atención sobre el hecho de que reposa sobre una premisa que no ha sido probada, es importante observar que la tesis a. es expresamente defendida por Frege en GA, en donde afirma que representaciones son aquello a lo cual se refieren las leyes de la asociación ${ }^{6}$.

\section{Decisiones y motivación}

Si se presta atención al pasaje que hemos considerado hasta ahora y se lo interpreta en el sentido en que lo hemos hecho, entonces no se pasará por alto dos textos, uno de la "Logik" de 1897 en el cual Frege observa que nuestros conocimientos producen (einwirken) nuestras decisiones ${ }^{7}$, otro de

6 "Die Vorstellung [...] ist das, worauf sich die psychologischen Assoziationsgesetzte beziehen; [...]" (GA § 27 (41-42)). Que el texto citado no es una raridad, sino que expresa una firme convicción, queda claro si lo comparamos con este otro de GGA: "Und daher kommt ein Schwanken in den Gebrauch des Wortes 'Vorstellung', indem es bald etwas zu bedeuten scheint, was dem Seelenleben des Einzelnen angehört und nach psychologischen Gesetzen mit anderen Vorstellungen verschmilzt, sich mit ihnen associirt, bald etwas Allen gleicherweise Gegenüberstehendes, bei dem ein Vorstellender weder genannt noch auch nur vorausgesetzt wird. Diese beiden Gebrauchsweisen sind unvereinbar; denn jene Associationen, Verschmelzungen, gehen nur im einzelnen Vorstellenden vor sich und gehen nur an etwas vor sich, was diesem Vorstellenden ganz so eigenthümlich zugehört, wie seine Freude oder sein Schmerz es thut". (GGA, XVIII). Obsérvese que en este texto las leyes psicológicas y las leyes de la asociación son tomadas como equivalentes. No hay ningún motivo para pensar que Frege acepte otras leyes psicológicas que no sean las de la asociación. Sea cual sea el caso, de todas formas, no se puede pasar por alto que las leyes de la asociación parecen ser el modelo 0 tipo básico de las leyes psicológicas.

7 "Wenn man von einer Wirklichkeit des Gedanken sprechen will, so kann dies nur in dem Sinne geschehen, dass die Kenntnis, die jemand, z.B. von einem Naturgesetz hat, auf seine Entschlüsse einwirkt, die dann wieder Massenbewegungen zur Folge haben können" (L (1897), 53).

Rev. Filos., Aurora, Curitiba, v. 25, n. 37, p. 289-303, jul./dez. 2013 
"Der Gedanken" en donde Frege nota que pensamientos actúan (wirken) a través del hecho de que se los capta y se los tiene por verdaderos ${ }^{8}$. En ambos textos se nos dice básicamente dos cosas, a saber, que

a) las decisiones son causadas;

b) y son causadas a través de un modo peculiar de causación. Analicemos ahora cada una de ellas.

\section{Libertad de indeterminación vs. razón suficiente}

Detengámonos por un momento a atender las peculiaridades de la teoría fregueana de la decisión y, eventualmente, una vez constatadas las mismas, preguntar por sus posibles motivaciones. Para tal propósito, comparemos lo que Frege efectivamente afirma con una eventual concepción alternativa. Se podría pensar que las decisiones son tomadas simplemente "porque sí", sin causa o razón alguna, o sea, podríamos pensar las decisiones, en el marco de una teoría de la libertad, como indeterminación. Ahora bien, Frege parece no suscribir tal teoría. Las decisiones no son absolutamente carentes de todo fundamento, sino que, al menos en un cierto sentido, ellas son "causadas" (eingewirkt). Frege parece, pues, inclinarse por una teoría de la libertad que respeta la validez irrestricta del principio de razón suficiente?.

8 "Wie wirkt ein Gedanke? Dadurch, dass er gefasst und für wahr gehalten wird. Das ist ein Vorgang in der Innenwelt eines Denkenden, der weitere Folgen in der Innenwelt haben kann, die, auf das Gebiet des Willens übergreifend, sich auch in der Aussenwelt bemerkbar machen. Wenn ich z.B. den Gedanken fasse, den wir im pythagoreischen Lehrsatze aussprechen, so kann die Folge sein, dass ich ihn als wahr anerkenne, und weiter, dass ich ihn anwende, einen Beschluss fassend, der Beschleunigung von Massen bewirkt. So werden unsere Taten gewöhnlich durch Denken und Urteilen vorbereitet. Und so können Gedanken auf Massenbewegungen mittelbar Einfluss haben" (G, 76-77(53)). Obsérvese que por razones puramente gramaticales, en vez de "Entschlüsse" Frege usa en este texto la expresión "Beschlüsse". Dejando de lado esta pequeña diferencia, estilística y no-sustancial, la idea expresada es la misma que en el texto anterior.

9 Aun cuando es absolutamente irrelevante para el objetivo central de mi texto, no deja de ser interesante observar que quizás esta concepción de libertad debe ser encuadrada en el marco de la discusión entre católicos y protestantes y, por tal razón, tal vez tenga su raíz última en la formación religiosa de Frege.

Rev. Filos., Aurora, Curitiba, v. 25, n. 37, p. 289-303, jul./dez. 2013 


\section{Decisión y motivación}

Ahora bien, si se puede decir que las decisiones, en un cierto sentido, son "causadas", se debe tomar extremo cuidado en precisar lo que se está entendiendo, en este caso, por "causación". "Causación" no significa aquí en modo alguno el ser "determinado" por leyes naturales o, en forma más precisa, el ser determinado a comportarse según leyes naturales, sino que significa el ser "motivado" por un saber ${ }^{10}$.

Frege está, pues, diferenciando dos tipos de causación (wirken) esencialmente heterogéneos, uno natural-causal y uno "intencional". Las decisiones son tomadas sobre la base de un saber y un saber es un estado intencional. Lo que diferencia los dos tipos de causalidad es, entonces, la diferencia entre una causalidad que envuelve estados intencionales y una que ocurre con absoluta independencia de ellos. Dicho más concretamente: existe una diferencia absoluta en el efecto que ejerce una piedra que cae sobre mi cabeza y mi decisión de cambiar de lugar, ya que sé lo que habrá de ocurrir, si la piedra cae encima de mi cabeza. No es el hecho de que la piedra va a caer sobre mi cabeza, sino mi saber a su respecto, lo que "motiva" mi decisión. Hechos o eventos determinan causalmente otros hechos o eventos; un saber acerca de un hecho o evento "motiva" una toma de decisión.

Si bien son muy pocos los textos en que Frege habla de "decisiones" (Entschlüsse, Beschlüsse), ellos, no obstante, manifiestan cosas sustanciales que, por otra parte, son totalmente coherentes entre sí e, incluso, se exigen de modo necesario. En realidad, la afirmación fregueana, de que las decisiones no se encuentran sometidas a las leyes de la asociación (y, con ello, al determinismo mecánico-natural) y la afirmación fregueana de que las decisiones son en realidad motivadas por un saber, no son otra cosa que las dos caras de una misma moneda, esto es, la formulación negativa y positiva de una

10 Una pregunta ulterior podría ser si tiene sentido pensar que toda decisión es motivada por un saber o si tenemos que llegar, en última instancia, a decisiones que no responden a motivación alguna. 
misma concepción fundamental. Negativamente, no son las leyes de la asociación las que determinan nuestras decisiones; positivamente, nuestras decisiones son motivadas por nuestro saber.

\section{Motivación e intersubjetividad: motivación como ley del mundo espiritual}

La línea demarcatoria entre motivación y causalidad no corre paralela a la línea demarcatoria entre mundo interno y externo. La motivación no solo está presente en el primero, sino también, en un cierto sentido, en el segundo. En efecto, inmediatamente a continuación del texto citado Frege observa, que la acción (Wirken) de un hombre sobre otro se produce la mayoría de las veces a través de la comunicación de pensamientos ${ }^{11}$.

Los seres humanos actúan sobre otros seres humanos, no de un modo causal-mecánico, sino "motivacional", esto es, seres intencionales son capaces de actuar sobre otros seres intencionales, no (meramente) en cuanto ejercen acciones que tienen efectos mecánicos sobre el otro, sino en cuanto efectúan acciones que motivan estados intencionales en otros seres intencionales. La causalidad de motivación no es, pues, solo esencial para comprender la vida del sujeto solipsístico, sino, asimismo, para dar cuenta del fenómeno de la intersubjetividad ${ }^{12}$.

Si hasta ahora habíamos introducido la noción de "motivación" sin que esta decisión terminológica tuviese asidero alguno en el texto fregueano, en el último pasaje referido, no obstante, Frege emplea la expresión "veranlassen". Ahora bien, "veranlassen" no es sino una de las

11 "Das Wirken von Mensch auf Mensch wird zumeist durch Gedanken vermittelt. Man teilt einen Gedanken mit. Wie geschieht das? Man bewirkt Veränderungen in der gemeinsamen Aussenwelt, die, von dem andern wahrgenommen, ihn veranlassen sollen, einen Gedanken zu fassen und inn für wahr zu halten. Die grossen Begebenheiten der Weltgeschichte, konnten sie anders als durch Gedankenmitteilung zustande kommen?" (G, 53(77)).

12 Obsérvese, y en esto vamos más allá del texto de Frege, que motivar la conducta de otro ser intencional no acontece meramente a través de la producción de signos. Un gesto, una conducta (tal como un simple abrazo) actúa a través de su significado y, en consecuencia, es "motivante". De lo anterior resulta una importante consecuencia: decíamos que la motivación supone estados intencionales. Podemos decir también (y esto no es menos esencial) que el factor "motivante" es en realidad siempre una "significación". La motivación es el modo de acción de las significaciones, que solo puede actualizarse a través de estados intencionales.

Rev. Filos., Aurora, Curitiba, v. 25, n. 37, p. 289-303, jul./dez. 2013 
formas germánicas en las cuales se expresa aquella idea que, en su raíz latina, se formula como "Motivation". "Motivieren" y "veranlassen" son hasta cierto punto sinónimos y, por tanto, intercambiables. En suma, hablar de una forma particular de causalidad como "Motivation" en Frege, tiene su justificación en el propio léxico fregueano.

\section{Motivación y comunicación}

Detengámonos ahora en un punto específico del texto anterior. En él están contenidas al menos dos relaciones de motivación diferenciables. Podemos decir que un ser humano "motiva" a otro ser humano a tomar una decisión o comportarse de una cierta manera en la medida en que transmite una información. No obstante, no podemos pasar por alto que la motivación no solo se da en la relación entre dos seres humanos y, eventualmente, con respecto al signo, sino también en la relación de cada ser humano, tomado aisladamente, con un signo. En efecto, es irrelevante si un determinado conjunto de signos está allí presente de un modo simplemente accidental o con el objetivo de provocar tal o cual conducta. Lo importante es que, sea cual sea el caso, la presencia del signo actúa sobre el sujeto que lo percibe "motivando" la aprehensión de un determinado pensamiento y no propiamente causando la misma de un modo mecánico, eventualmente, a través de una asociación.

Que Frege no piensa la comunicación como un fenómeno explicable en términos meramente asociativos, sino como un fenómeno que envuelve de modo esencial un elemento intencional y comprende un modo de causación específico, la motivación, es un hecho que ha sido pasado por alto por los críticos, los cuales tienden a presuponer sin más que Frege "tiene" que explicar la comunicación en términos puramente asociativos ${ }^{13}$. Sobre esta base es usual, ulteriormente, desarrollar una crítica a las pretendidas insuficiencias de la teoría fre-

13 Lasleyes de la asociación solo pueden tener lugar entrelas representaciones. Las representaciones (y solo las representaciones) pueden ser asociadas. Si esto es así, entonces no tiene sentido decir que una representación o, eventualmente, un signo es asociado a un determinado pensamiento (Gedanke).

Rev. Filos., Aurora, Curitiba, v. 25, n. 37, p. 289-303, jul./dez. 2013 
gueana de la comunicación. Todo esto resposa, no obstante, sobre un supuesto erróneo que contradice una lectura más atenta del texto fregueano, aun cuando, tal vez, no da solución a todos los problemas que ya han sido apuntados.

\section{Decisiones, asociación y motivación: la reafirmación de la lectura intencional}

Ya observamos que la intencionalidad es la condición necesaria de la causalidad por motivación. Solo seres que poseen estados intencionales son capaces de desenvolver una causalidad por motivación. Ahora bien, la admisión de una "causalidad motivacional" es una prueba suplementaria de que Frege tiene una concepción intencional y no-naturalística de la conciencia. Dicho de otra forma: precisamente porque Frege tiene una concepción intencional de la conciencia y no una naturalística, posee, asimismo, una concepción motivacional y no puramente mecánica de su causalidad.

Una característica esencial de la relación intencional, que la diferencia de toda relación real, es que uno de sus elementos puede no existir. Ahora, este es justamente un rasgo decisivo en la causalidad por motivación. Es inherente a una relación motivacional, el que uno de sus elementos, a saber, la causa, puede no ser real. Mi conducta es determinada por mi creencia, independientemente del hecho de que ella sea verdadera o falsa y de que una realidad corresponda o no a la misma. Aun cuando yo me equivoque y sea falso que delante de mí se encuentra un animal peligroso que me puede atacar, el hecho de que yo así lo creo, es determinante en mi decisión de huir.

\section{Frege y Husserl, sobre la subjetividad}

Como hemos visto, existen razones en el propio texto fregueano que legitiman hablar de un tipo peculiar de causalidad por 
“motivación". Existe, sin embargo, otra razón histórico-filosófica y sistemática más importante y que es exterior al texto. La noción de "motivación", como es sabido, es introducida por Husserl (1952) en "Ideas II" (§ 54s.) para referirse a un modo de causalidad peculiar del mundo espiritual. Conviene entonces detenernos en esta noción husserliana y efectuar algunas observaciones.

Nuestra oposición a la lectura estándar de Frege nos ha llevado a oponernos, asimismo, a la lectura estándar de su relación con Husserl. En tal sentido, hemos insistido en que la diferencia entre Frege y Husserl no es que el primero no tenga ningún interés por la cuestión de la subjetividad, el segundo la haga su tema central, o que el primero defienda una concepión naturalista y no intencional de la conciencia, el segundo no. Tanto Frege como Husserl defienden una concepción intencional de la conciencia y la verdadera diferencia radica en el modo en que esta intencionalidad es concebida, o sea, en el hecho de que en Husserl, pero no en Frege, es pensada en el marco del principio de inmanencia, aun cuando, a partir de 1906, con la doctrina de la reducción, se efectúe una significativa reformulación de dicho principio ${ }^{14}$. Ahora, el hecho de que ambos autores coincidan en asumir una teoría intencional de la subjetividad es coherente con (y confirmada por) el hecho de que ambos consideren un tipo de causalidad específica a la misma. Frege no solo coincide, pues, con Husserl en defender una concepción intencional y no-naturalística de la conciencia, sino también, y justamente por lo anterior, en poseer una concepción motivacional y no mecánicodeterminista de su causalidad ${ }^{15}$.

Mas aún, no solo hay en Frege la afirmación genérica de una causalidad en cuanto motivación, sino que, más allá de lo anterior, y como

14 En otro artículo específico nos proponemos efectuar de forma extensa una comparación entre las posiciones de Frege y Husserl que, como ya es de esperar, se aparta radicalmente de la que se ha tornado usual. Además del punto de contacto que indicamos ahora, tiene que ser recordada la teoría de la percepción. Pero no se trata de afirmar solo puntos de contacto y, eventualmente, la existencia de una identidad entre las posiciones de ambos, sino también, más correctamente, de mostrar, sobre la base de los puntos de contactos, las diferencias decisivas. Ellas son básicamente dos, a saber, además de la ya mencionada concepción de intencionalidad, el hecho de que Frege no tiene una justificación metódica de sus afirmaciones respecto de la subjetividad, justificación metódica que, por el contrario, es central en Husserl.

15 No está de más observar que esta idea aparece en Frege en 1897. 
en Husserl, la misma se proyecta de modo esencial en su concepción de las relaciones intersubjetivas y en el modo de concebir la comprensión del signo. También en estas cuestiones más específicas tenemos puntos de contacto entre Frege y Husserl, dado que también Husserl pretende ofrecer una concepción no-asociacionista e intencional de la comunicación, no menos que de las relaciones intersubjetivas en general.

Lo dicho no implica, de ningún modo, afirmar que en Frege están presentes todos los elementos de la idea de motivación de Husserl. En efecto, podríamos hacer una larga lista de diferencias sobre la base de las coincidencias básicas. A modo de ejemplo, dos sean aquí apuntadas:

a) Para Frege, la causalidad por motivación parece caracterizar la relación de ciertos estados intencionales pero, en ningún momento, como en Husserl, Frege cuestiona de principio la idea de asociación o intenta reinterpretarla. En Frege, la causalidad por motivación y por asociación coexisten, siendo que solo entre algunos estados intencionales se establece una relación "motivacional". En Husserl, por el contrario, la vida realmente psíquica es siempre "motivada", debiéndose incluso reformular la idea de asociación sobre la base de la misma.

b) Por otra parte, todo aquello que en Husserl vincula la teoría de la motivación a la reducción y al punto de vista de la fenomenología transcendental está ausente en Frege, quien en ningún momento podría acompañar este movimiento, ya que cuestiona su presupuesto básico, o sea, el principio de inmanencia.

Se dice muchas veces al respecto de victorias militares o deportivas, que las mismas dependen de detalles. También teorías científicas e, incluso, interpretaciones de obras y autores dependen de detalles. Cada detalle, tomado aisladamente, es insignificante. En su conjunto, no obstante, ellos se tornan significativas y la atención a los mismos permite descubrimientos relevantes.

Mi interpretación de Frege se basa, por un lado, en claros e inequívocos textos, que son absolutamente explícitos y transparentes en 
sus propósitos, como es el caso de todos aquellos en que se niega, de una forma u otra, el principio de inmanencia. Ella se basa, asimismo, en varios otros, que han sido pasados por alto y entre los cuales no se veía relación alguna. Entre ellos pueden ser ennumerados ${ }^{16}$ :

1) la crítica de Kerry a Frege;

2) el pasaje de GGA, en donde Frege observa que no lucha contra molinos de viento;

3) la estrategia de GGA de clarificar la propia posición en contraposición con la opuesta;

4) la estrategia de GGA en el sentido de buscar el origen de la discusión con el psicologismo;

5) el pasaje de la "Lógica" de 1897 en donde Frege se coloca la objeción de que, por lo menos, la captación del pensamiento es algo psicológico;

6) el pasaje del mismo texto en que se apunta a que la derivación de lo objetivo, a partir de lo subjetivo, solo puede dar cuenta de la apariencia de la objetividad;

7) la diferencia existente entre la crítica del psicologismo en GA y en GGA, concentrada en la primera en el empirismo, en la segunda en el idealismo y;

8) la nota de pie de página de la "Lógica” de 1897.

A esta lista debemos agregar los textos sobre decisiones y motivación que hemos analizado en este artículo. Que cosas aparentemente inconexas y en sí insignificantes, muestren de repente una relación entre sí y, a través de ella, devengan relevantes, es un elemento decisivo a favor de la prueba de una determinada hipótesis hermenéutica, no menos que históricamente lo ha sido en la física.

Debemos finalizar este artículo apuntando a una nueva cuestión $\mathrm{y}$, con ello a la necesidad de un ulterior complemento ${ }^{17}$. Los

16 Véase al respecto del autor: "La evolución de la crítica fregeana al psicologismo” (GONZÁLEZ PORTA, 2012a),"Horror subjectivi. La polémica entre Kerry y Frege en torno al método psicológico" (de próxima aparición), "La lógica fregueana de 1897 y su réplica al psicologismo" (de próxima aparición).

17 Véase GONZÁLEZ PORTA, $2012 b$. 
textos citados se encuentran en un contexto que ha servido a Currie (1982) como base para una interpretación radicalmente contraria a la aquí propuesta, según la cual, en última instancia, la aprehensión de los pensamientos supone de algún modo que ellos actúan sobre la subjetividad de modo causal. Ciertamente, el mencionado contexto tiene que ser considerado. Si se lo hace, no obstante, nos vemos obligados a llegar a una conclusión radicalmente diferente de la de Currie (1982), quien, por otra parte, pasa absolutamente por alto los elementos aquí analizados.

\section{Referencias}

CURRIE, G. Frege: an introduction to his philosophy. Sussex: The Harvester Press; New Jersey: Barnes Nobles Books, 1982.

FREGE, G. Grundgesetze der Arithmetik. Begriffschriftlich abgeleitet. I. Band. Jena: H. Pohle, 1983.

FREGE, G. Der Gedanke. In: Logische Untersuchungen. 3 Aufl. Göttingen: Vandenhoeck; Reihe, 1986.

FREGE, G. Die Grundlagen der Arithmetik. Hamburg: Meiner, 1988.

FREGE, G. Logik. In: Schriften zur Logik und Sprachphilosophie. Aus dem Nachlass. 3 Aufl. Hamburg: Meiner, 1990. p. 35-73.

GONZÁLEZ PORTA, M. A. La cuestión noética en Frege, su concepto de intencionalidad y su influencia sobre Husserl. Thémata: Revista de Filosofía, n. 24, p. 83-114, 2000.

GONZÁLEZ PORTA, M. A. La evolución de la crítica fregueana al psicologismo. Veritas, v. 57, n. 2, p. 99-122, 2012a.

GONZÁLEZ PORTA, M. A. Currie y la interpretación literal de la tesis fregueana de la eficacia de pensamientos. Cognitio, v. 13, n. 2, p. 301-318, 2012b.

Rev. Filos., Aurora, Curitiba, v. 25, n. 37, p. 289-303, jul./dez. 2013 
HUSSERL, E. Ideen zu einer reinen Phänomenologie und phänomenologische Philosophie. Zweites Buch. Phänomenologische Untersuchungen zur Konstituition. Haag: Martinus Nijhoff, 1952.

KÜNNE, W. Die Philosophische Logik Gottlob Freges. Ein Kommentar. Frankfurt am Main: Klostermann, 2010.

Recibido: 10/07/2012

Received: 07/10/2012

Aprobado: 05/07/2013

Approved: 07/05/2013 